\title{
December 2017 Critical Care Case of the Month
}

\author{
Michael B. Gotway, MD \\ Department of Radiology \\ Mayo Clinic Arizona \\ Scottsdale AZ USA
}

Clinical History: A 57-year-old man with no known previous medical history was brought to the emergency room via ambulance and admitted to the intensive care unit with a compliant of severe chest pain in the substernal region and epigastrium. The patient was awake and alert and did not complain of shortness of breath.

Physical examination was largely unremarkable and the patient's oxygen saturation was $98 \%$ on room air. The patient's vital signs revealed tachycardia (105 bpm) and his blood pressure was $108 \mathrm{mmHg} / 60 \mathrm{mmHg}$.

Laboratory evaluation showed a slightly elevated white blood cell count $\left(13 \times 10^{9}\right.$ cells/L), but his hemoglobin and hematocrit values were with within normal limits, as was his platelet count.

Which of the following diagnoses are appropriate considerations for this patient's condition?

1. Acute pericarditis

2. Aortic dissection

3. Community-acquired pneumonia

4. Myocardial infarction

5. All of the above 


\section{Correct! \\ 5. All of the above}

All of the conditions listed above could produce chest pain and result in a mildly increased white blood cell count accompanied by tachycardia and mild hypotension. One may expect a complaint of shortness of breath if community-acquired pneumonia were responsible for the patient's presentation, but community-acquired pneumonia is a common condition and the degree of shortness of breath that may occur in patients with acute respiratory infections may vary considerably.

Which of the following represents the most appropriate next test to obtain for the evaluation of this patient?

1. Chest radiography

2. Echocardiography

3. Electrocardiogram

4. 1 and 3

5. 2 and 3 


\section{Correct!}

\section{1 and 3}

Myocardial infarction is a leading differential diagnostic consideration for the presentation of this patient, and electrocardiography is an important first step in the assessment of this possible condition. Chest radiography is also an important first step in the evaluation of this patient because community-acquired pneumonia is also a leading consideration for this patient's presentation. Furthermore, when myocardial infarction is a possibility, another leading cause of acute chest pain- aortic dissectionmust also be considered and excluded, and chest radiography is often used to assess for the widened mediastinum that may herald the presence of this disorder.

Echocardiography is not an unreasonable test to obtain for this patient, particularly given the possibility of pericarditis as a cause of the patient's presenting complaint of chest pain, but it may be more rewarding to obtain the electrocardiogram first and then base the next steps on the result of the electrocardiogram and chest radiographic results before proceeding to echocardiography, particularly given that no clinical evidence of heart failure was noted.

The patient's electrocardiogram showed mild tachycardia with an otherwise normal rhythm. The patient's first troponin level was normal. Frontal chest radiography was performed (Figure 1).

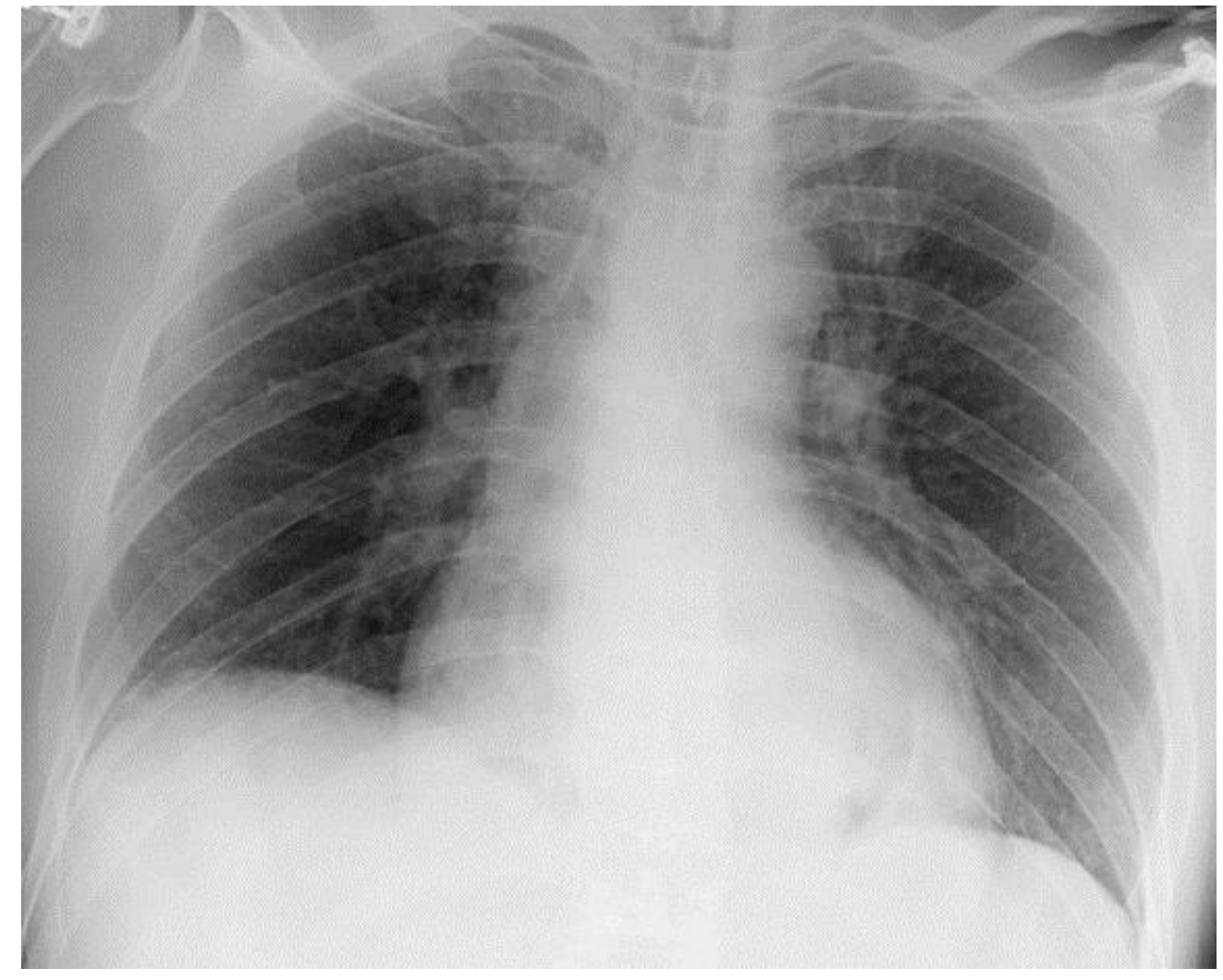

Figure 1. Frontal chest radiography. 
Which of the following represents the most accurate assessment of the chest radiographic findings?

1. The frontal chest radiograph shows "Westermark's sign" suggesting acute pulmonary embolism

2. The frontal chest radiograph shows focal consolidation suggesting pneumonia

3. The frontal chest radiograph shows no specific abnormalities

4. The frontal chest radiograph shows pleural effusion

5. The mediastinum appears abnormally wide, suggesting an acute aortic syndrome 


\section{Correct! \\ 3. The frontal chest radiograph shows no specific abnormalities}

The frontal chest radiograph shows slight widening of the mediastinum with a prominent aortic arch, but this appearance is well within the normal range for a portable, frontal chest radiograph. No specific features to suggest an acute aortic syndrome are present, but it is well-recognized that acute aortic syndromes often present with few or nonspecific findings at chest radiography, and chest radiographs may even be normal in this condition. No focal consolidation or pleural effusion are present. The right lung appears relatively "hyperlucent" compared to the left lung, which could be interpreted as Westermark's sign. Westermark's sign represents decreased pulmonary vascularity due to mechanical obstruction or reflex vasoconstriction due to pulmonary embolism. This chest radiograph sign is very non-specific and typically the hyperlucency that accompanies this sign is far more commonly caused by technical considerations, such as patient rotation, or obstructive lung disease, rather than acute pulmonary embolism. The chest radiograph shown in Figure 1 shows slight left anterior oblique rotation, which is likely the cause of the relative hyperlucency of the right hemithorax. The pulmonary vascularity is not grossly asymmetrically decreased on the right, nor is the right hilum noticeably enlarged, as may occur with acute pulmonary embolism, and therefore Westermark's sign is not an appropriate description for the appearance of the chest radiograph shown in Figure 1.

The patient's d-dimer level was within the normal range.

Which of the following statements is the most appropriate next step for the management of this patient?

1. ${ }^{133}$ Xe-Ventilation - ${ }^{99 m}$ Tc-perfusion scintigraphy

2. Chest CT

3. Echocardiography

4. Repeat frontal and lateral chest radiography

5. Thoracic MRA 


\section{Correct! \\ 2. Chest CT}

Chest CT is probably the next most appropriate test to obtain among the choices listed. The troponin and electrocardiogram results make myocardial infarction unlikely in this patient. Pulmonary embolism is also unlikely given the lack of shortness of breath, the relatively good room air oxygenation, and the negative d-dimer results. An acute aortic syndrome remains a possibility, as does pericarditis, and even pneumonia, while less likely given the relatively normal appearance of the frontal, portable, chest radiograph, remains a consideration, and all of these diagnoses can be readily visualized at chest $\mathrm{CT}$. Both thoracic MRA and echocardiography would prove useful for the assessment of pericarditis and are also useful tests for acute aortic syndromes, although chest CT is preferred for the latter.

Chest CT was ordered. The patient was awake and alert, still complaining of severe chest and epigastric pain, and further questioning was performed while awaiting chest CT. The patient admitted to recent upper gastrointestinal illness which was accompanied by significant nausea and vomiting. The patient had not vomited since his arrival in the emergency room, but did undergo severe retching with his vomiting prior to the development of his chest pain and calling for the ambulance.

Which of the following represents a new important differential diagnostic consideration for this patient's presentation?
1. Empyema
2. Esophageal rupture
3. Lobar pulmonary torsion
4. Pneumothorax
5. Thoracic spine discitis 


\section{Correct! \\ 2. Esophageal rupture}

The information regarding a recent upper gastrointestinal illness leading to severe bouts of vomiting with retching, followed by chest pain, specifically suggests the possibility of esophageal rupture. Thoracic spine discitis would be expected to cause back pain, possibly with fever, typically in the context of an infectious source elsewhere that leads to bacteremia seeding of the bloodstream. Occasionally discitis and spinal osteomyelitis could be caused by more indolent infections, such as Mycobacterium tuberculosis or endemic fungi, but the patient's pain would still more likely be posteriorly located, not epigastric and substernal in location. The pain associated with symptomatic pneumothorax would more likely be lateralizing and accompanied by shortness of breath, and the pneumothorax would likely have been visualized at frontal chest radiography. Similarly, chest discomfort associated with empyema would also more likely lateralize, and the pleural abnormality associated with this condition should be visible at chest radiography. Torsion of a lobe of the lung is an exceedingly rare condition that typically occurs following thoracic surgery and lobectomy, which is not a relevant consideration for this patient. Furthermore, chest radiographs are typically abnormal in patients with pulmonary torsion, showing areas of consolidation. Such findings are non-specific, but are nevertheless entirely lacking on this patient's frontal chest radiograph.).

Given the above information, which of the following represents the most appropriate next step for the management of this patient?

1. Abdominal CT

2. Barium esophagram

3. Cardiac MRI

4. Chest CT

5. Upper endoscopy 


\section{Correct! \\ 4. Chest CT}

Upper endoscopy may ultimately be required for this patient if esophageal injury is suggested, but typically that diagnosis is first approached non-invasively. Cardiac MRI would be of value for assessment of cardiac function, possible pericardial disease, and even myocardial and aortic diseases, but the recently obtained historical information points more towards an esophageal etiology, and therefore cardiac MRI would not be the ideal test choice. Abdominal CT may show distal esophageal abnormalities and abnormalities involving the lung bases and inferior pleural space, but most thoracic causes for this patient's presentation would only be partially visualized on abdominal CT and further imaging of the thorax would still be required. An esophagram is an appropriate test for this patient in light of the recently obtained historical information, but, when esophageal perforation is suspected, typically water-soluble oral contrast, not barium is employed, and therefore choice " 3 " is not the best choice.

Which of the following represents the optimal chest CT protocol for this patient?

1. CT aortography

2. CT pulmonary angiography

3. Intravenously contrast-enhanced chest CT followed by repeat CT using orally administered intravenous contrast

4. Lateral decubitus CT

5. Unenhanced chest CT 


\section{Correct! \\ 3. Intravenously contrast-enhanced chest $\mathrm{CT}$ followed by repeat $\mathrm{CT}$ using orally administered intravenous contrast}

Any form of CT- unenhanced or enhanced with injection of intravenous contrast using either an aortography or pulmonary angiography protocol- would be useful for assessment of this patient, but employing a CT protocol specifically tailored to assess for esophageal injury that can simultaneously assess the lungs, aorta, and pleural space would be the optimal approach. Such a protocol initially employs intravenously enhanced CT imaging (without oral contrast) to detect pneumomediastinumesophageal injury should show this finding, and the absence of pneumomediastinum should exclude significant esophageal perforation. Additionally, this protocol can simultaneously evaluate the aorta, lungs, and pleural space for the various alternative causes of chest pain that could clinically simulate esophageal injury. If no pneumomediastinum is detected, further imaging is not required. If pneumomediastinum is found, then oral contrast is administered. Generally when esophageal injury is present, water-soluble contrast media is utilized in the event perforation has occurred; the use of barium in this situation is generally avoided owing to fears of the potential development of granulomatous mediastinitis if the barium contrast material gains access to the mediastinum. However, the commonly employed water-soluble contrast agent used for upper gastrointestinal examinations is hyperosmolar, and inadvertent aspiration of this substance can lead to pulmonary edema. Therefore, diluted intravenous contrast material is an excellent oral contrast agent to employ when using CT to assess for esophageal injury because the contrast agents used for intravenous contrast injections cause little reaction if aspirated and are generally inert should they reach the mediastinal tissues. This dilute, orally administered intravenous contrast material is given just prior to a separate second CT acquisition- if the pneumomediastinum seen on the first acquisition fills with the orally administered contrast agent, the diagnosis of esophageal perforation is established. Lateral decubitus CT may show findings suggesting esophageal injury, namely pneumomediastinum- but the lateral decubitus position is not of particular benefit for esophageal assessment. Lateral decubitus CT has been suggested as a useful technique to demonstrate air trapping [in the dependent lung] for patients who cannot properly cooperate with the breathing instructions required for supine post-expiratory imaging.

The patient underwent chest CT using the specific esophageal protocol described above (Figure 2). 

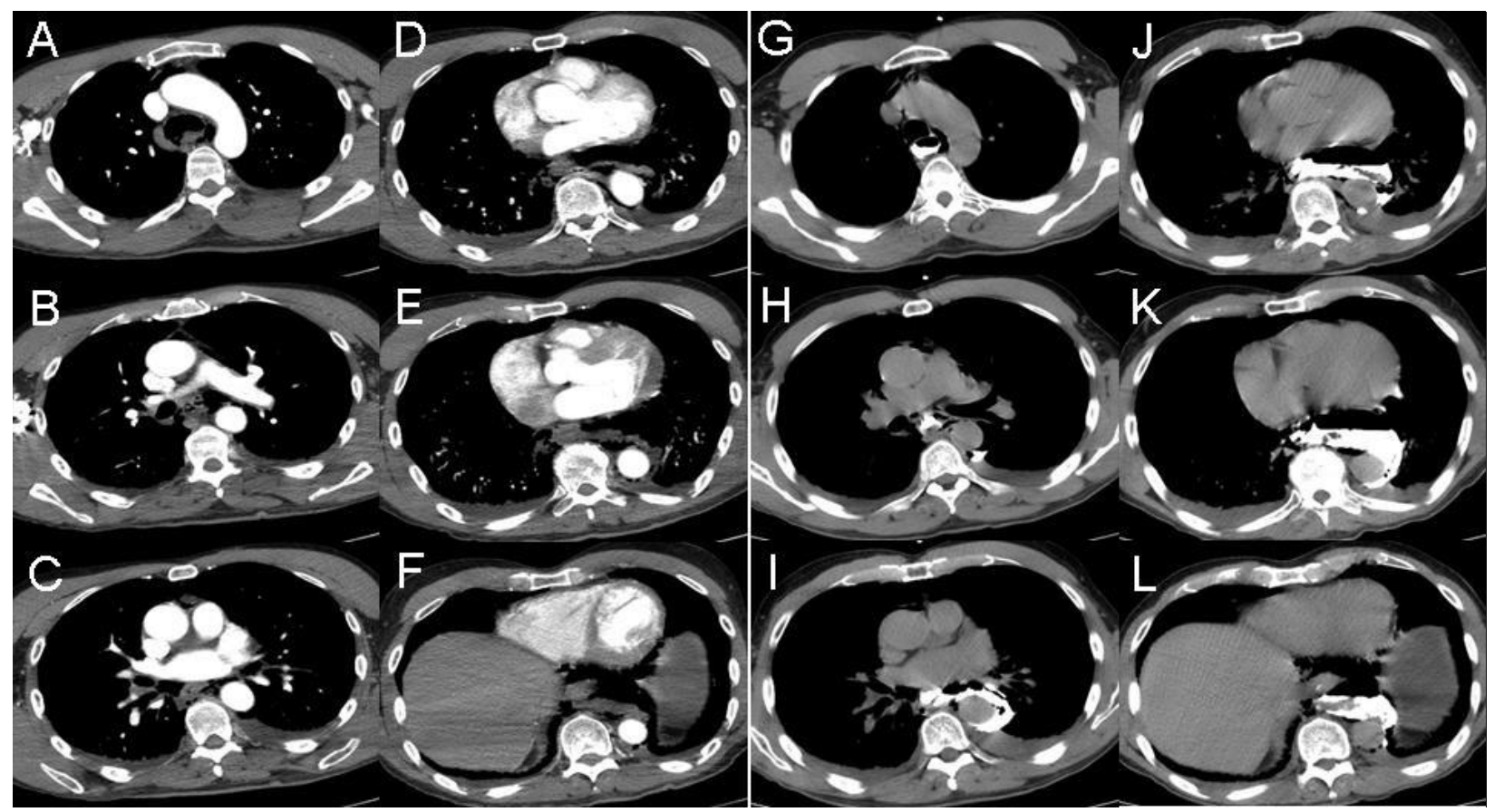

Figure 2. A-F: Representative images from the CT esophagram following the intravenous injection of contrast material but prior to the oral administration of dilute intravenous contrast material. G-L: Representative images from the CT esophagram following the oral administration of dilute intravenous contrast material.

Which of the following represents the most accurate assessment of the chest CT findings?

1. The chest CT shows acute pulmonary embolism

2. The chest CT shows aortic dissection

3. The chest CT shows empyema

4. The chest CT shows pericarditis

5. The CT findings are diagnostic of esophageal rupture 


\section{Correct!}

\section{The CT findings are diagnostic of esophageal rupture}

The unenhanced phase of the chest CT examination shows pneumomediastinum clustering in proximity to the esophagus. On the second, oral contrast administration phase, the areas of pneumomediastinum clearly fill with oral contrast, indicating the presence of esophageal perforation (Figure 3).
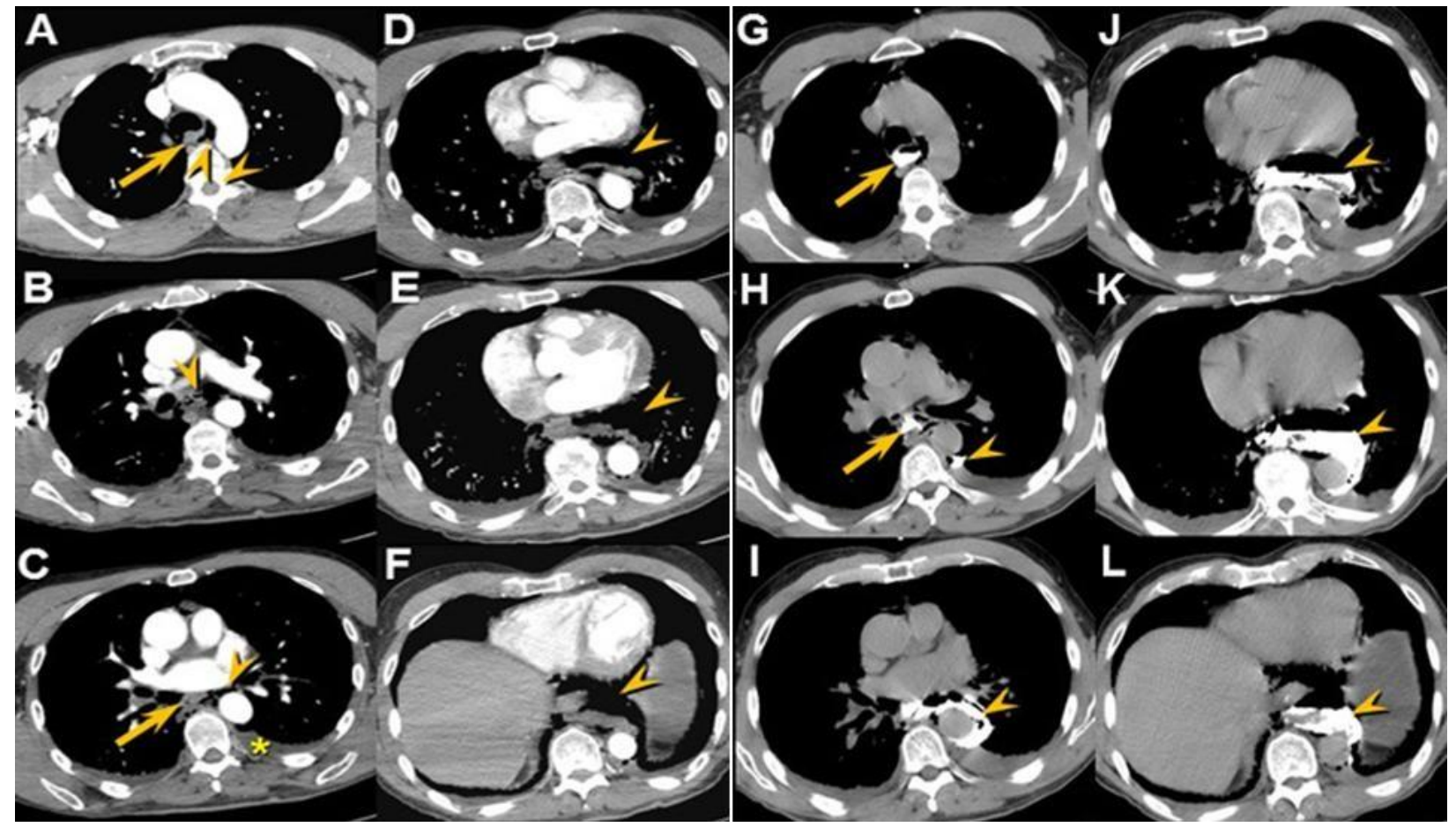

Figure 3. A-F: CT esophagram following the intravenous injection of contrast material shows pneumomediastinum (arrowheads) clustering around the esophagus

(decompressed soft tissue structure indicated by arrow). A small left pleural effusion (*) is present. No evidence of aortic pathology or pulmonary embolism is seen. G-L: CT esophagram following the oral administration of dilute intravenous contrast material shows the pneumomediastinum clustering around the esophagus detected on the first

phase of the examination (A-F) fills with contrast on this phase of the examination (arrowheads), indicating esophageal rupture. Arrows= esophagus filled with orally administered contrast.

No evidence of pulmonary embolism or aortic dissection is present. The pericardium appears normal. Pleural effusion is present on the left, but the effusion is small, without pleural thickening, and the CT appearance does not suggest empyema.

The patient underwent surgical repair and recovered uneventfully.

Diagnosis: Esophageal rupture (Boerhaave's syndrome) 


\section{References}

1. Liguori C, Gagliardi N, Saturnino PP, Pinto A, Romano L. Multidetector computed tomography of pharyngo-esophageal perforations. Semin Ultrasound CT MR. 2016;37(1):10-5. [CrossRef] [PubMed]

2. Suarez-Poveda T, Morales-Uribe $\mathrm{CH}$, Sanabria A, Llano-Sánchez A, ValenciaDelgado AM, Rivera-Velázquez LF, Bedoya-Ospina JF. Diagnostic performance of CT esophagography in patients with suspected esophageal rupture. Emerg Radiol. 2014; 21(5):505-10. [CrossRef] [PubMed]

3. Fadoo F, Ruiz DE, Dawn SK, Webb WR, Gotway MB. Helical CT esophagography for the evaluation of suspected esophageal perforation or rupture. AJR Am J Roentgenol. 2004;182(5):1177-9. [CrossRef] [PubMed]

4. Ba-Ssalamah A, Zacherl J, Noebauer-Huhmann IM, Uffmann M, Matzek WK, Pinker K, Herold C, Schima W. Dedicated multi-detector CT of the esophagus: spectrum of diseases. Abdom Imaging. 2009;34(1):3-18. [CrossRef] [PubMed]

5. Corns RA, Edwards JL. Boerhaave's syndrome: CT diagnosis. Ann R Coll Surg Engl. 2004;86(6):W8-9. [CrossRef] [PubMed]

6. Carrott PW Jr, Low DE. Advances in the management of esophageal perforation. Thorac Surg Clin. 2011 Nov;21(4):541-55. [CrossRef] [PubMed] 